\title{
Oculomotor correlates of context-guided learning in visual search
}

\author{
YUAN-CHI TSENG and CHIANG-SHAN RAY LI \\ Chang Gung Memorial Hospital, Tao-Yuan, Taiwan
}

\begin{abstract}
Previous studies have shown that context-facilitated visual search can occur through implicit learning. In the present study, we have explored its oculomotor correlates as a step toward unraveling the mechanisms that underlie such learning. Specifically, we examined a number of oculomotor parameters that might accompany the learning of context-guided search. The results showed that a decrease in the number of saccades occurred along with a fall in search time. Furthermore, we identified an effective search period in which each saccade monotonically brought the fixation closer to the target. Most important, the speed with which eye fixation approached the target did not change as a result of learning. We discuss the general implications of these results for visual search.
\end{abstract}

It has been proposed that visual search proceeds in parallel and serial mechanisms. In a parallel mechanism, the target with a unique feature (such as color, orientation, size, form, luminance, or abrupt onset) "pops out," resulting in very rapid search behaviors (Cave, 1999; Jonides \& Yantis, 1988; Treisman \& Gelade, 1980; Turatto \& Galfano, 2000; Wolfe, 1994; Yantis \& Jonides, 1996). The search time required in order to identify the target is independent of the number of distractors. A serial mechanism, on the other hand, is employed when the target and distractors differ very little in physical characteristics. For instance, to search for a target "T" among distractors "Ls" requires an inspection of each item on the scene. The search time increases linearly with the number of distractors, and the search usually terminates after approximately half of the items have been inspected (Wolfe, 1994). A search task involving a serial mechanism is indeed more complex and harder to perform than one involving a parallel mechanism.

Searching for a target in a natural environment can be more complicated than doing so in a laboratory setting. Studies have shown that invariant visual context is an important resource in guiding spatial attention and facilitating visual search (Chun \& Jiang, 1998, 1999; Schneider \& Shiffrin, 1977; Shiffrin \& Schneider, 1977; and see Chun, 2000, for a review). Quite interestingly, a contextguided visual search appears to involve implicit learning (Chun, 2000; Chun \& Jiang, 1998, 1999; Olson \& Chun,

This study was supported by Grant CMRP792 from the Chang Gung Memorial Hospital. Y.-C.T. was supported by a research training grant from the National Science Council of Taiwan. We thank Su-Ling Yeh and I-Ping Chen for their discussions, Glyn Humphreys for his comments on an earlier draft, and Matthew Peterson, Richard Abrams, and two anonymous reviewers for their comments on the manuscript. Correspondence should be addressed to C.-S. R. Li, Connecticut Mental Health Center, Room S103, Department of Psychiatry, Yale University, 34 Park Street, New Haven, CT 06519 (e-mail: chiang-shan.li@yale.edu).
2001). In other words, although one is not consciously aware of the invariant context in which the target is imbedded, one benefits from the global cue while searching for the target. Chun and Jiang (1998) have suggested that contextual cuing is an example of instance-based learning: The presentation of a repeated context automatically activates learned instances of attentional guidance. They demonstrated that contextual priming reflects an effect of learning the global spatial layout rather than the lowlevel image properties of the visual items composing the context. Moreover, by studying the contextual search with different set sizes, they demonstrated a reduction in target search slope but not in intercept times. This latter finding suggested that contextual cuing increases the efficiency of search by guiding visual attention toward the target location rather than by facilitating other search processes such as initial perceptual processing of the displays or later motor response selection and execution (Chun \& Jiang, 1998).

In the above studies, the invariant visual context is composed of distractors, which are nonsalient. In real life, one's visual environment abounds with conspicuous objects, which automatically attract attention. For a timelimited search of a target, we may have to make an effort to ignore these distractors or to inhibit further devotion of our processing resources to these salient stimuli. An important question thus arises: Do we nevertheless take advantage of these salient distractors in our search of the target, while they are denied access to a higher level of representation? This issue is particularly interesting because it stands in contrast to our explicit use of visual landmarks in the surroundings to remember and to locate a target. The present study investigates this issue.

A concurrent goal of the present study is to understand the psychological processes underlying context-guided learning in visual search. Studies on visual search have traditionally relied on the analysis of reaction time to evaluate search behaviors (Treisman \& Gormican, 1988; 
Wolfe \& Cave, 1990; and see Wolfe, 1998, for a review). Since reaction time represents the overall outcome of the search task, which involves a myriad of neural and psychological mechanisms, it falls short of providing useful information about how context-guided learning takes place. Indeed, it has been shown that search behaviors involving different numbers of saccades and durations of fixations could result in the same response time in a search task (Zelinsky \& Sheinberg, 1995). It has been suggested that monitoring eye movements could provide important information about the mechanisms underlying different search behaviors. For instance, it was shown that parallel-serial search dichotomies were reflected in oculomotor behaviors (Zelinsky \& Sheinberg, 1997); semantic informativeness increased eye fixation density in a scene or visual search task (Henderson \& Hollingworth, 1999); and observers relied on learned regularities in the environment to schedule their searches (Shinoda, Hayhoe, \& Shrivastava, 2001).

Peterson and Kramer (2001a) employed eye movement monitoring to investigate how contextual information affected attentional guidance in visual search. Using a paradigm adapted from Chun and Jiang (1998), they found that fewer fixations were required to find the target when the subjects viewed the repeated stimulus configuration. Moreover, in some trials the attention guidance mechanism was able to precisely locate the target, such that the eyes went directly to the target when it was embedded in the repeated configuration. In the present study, we also investigated the patterns of eye movements as subjects learned to use contextual cues to facilitate the search of a target. Among the several oculomotor parameters we examined, we likewise found that a decrease in the number of eye fixations correlated with search performance.

The present study differs from Peterson and Kramer's work in several important aspects. First, the display configuration employed in our study is different. In Peterson and Kramer's (2001a) experiment, subjects searched for a target $\mathrm{T}$ among a number of distractors Ls, which could compose a visual context, repeatedly presented over trials. In the present study, subjects also searched for a $\mathrm{T}$ among Ls. However, the contextual cue was not composed of Ls, but of blue disks that physically did not resemble $\mathrm{T}$ or L. These items appeared visually salient in the display. We showed that subjects nevertheless learned to locate the target faster without explicit knowledge of the contextual cue. Second, we investigated oculomotor parameters, other than saccade number, that might index the contextual effect. Finally, we characterized the temporal dynamics of the search process and uncovered two search phases. The delineation of the two distinct phases of the search process allowed us to test alternative models of how contextual cuing might result in implicit learning during visual search. We showed that facilitation appeared to occur as a result of the earlier switch from an ineffective to an effective search phase, in which the distance between each successive eye fixation and the target declined monotonically.

\section{METHOD}

\section{Subjects}

Twelve healthy undergraduates from Chang Gung University, 18-25 years of age, were paid to participate in the experiment. All had normal or corrected-to-normal vision and were naive about the purpose of the experiment. None of the subjects had a history of neurological insult or use of illicit substances. Informed consent was obtained from all the subjects before the experiments were conducted, according to institute guidelines.

\section{Experimental Setup and Visual Display}

The experiment was carried out in a dark room. The subjects sat $50 \mathrm{~cm}$ in front of a ViewSonic P810 monitor that displayed the visual stimuli. Eye movement was monitored at $250 \mathrm{~Hz}$ and with a spatial resolution of $0.1^{\circ}$ by a video-based eyetracker (Eyelink, SR Research, Toronto) and corrected for head movements. Saccades satisfied a minimum velocity of $30 \mathrm{deg} / \mathrm{sec}$ and minimum acceleration of $9,500 \mathrm{deg} / \mathrm{sec}^{2}$. The saccade onset was defined as the time when the eye movement velocity exceeded $30 \mathrm{deg} / \mathrm{sec}$.

The visual display consisted of a target $\mathrm{T}$, distractors Ls, and cue elements, which could form a contextual (or cued) or random array. Each $\mathrm{T}$ and $\mathrm{L}$ subtended $0.98^{\circ} \times 0.14^{\circ}$ in visual angle, and both were light-gray (color 7, 16-color DOS VGA mode) and $3.81 \mathrm{~cd} / \mathrm{m}^{2}$ in luminance. There were two different target Ts - one with the horizontal arm pointing right and the other pointing left. After some preliminary experiments, we chose a more difficult configuration of the target T, with the length of one end of the top bar approximately $1 / 5$ of the other end. The cue element was a light-blue (color 9) disk with a diameter of $1.12^{\circ}$ and $4.73 \mathrm{~cd} / \mathrm{m}^{2}$ in luminance. All visual stimuli were presented against a dark (color $0, \approx 0.2 \mathrm{~cd} / \mathrm{m}^{2}$ ) background. There were eight blue disks in each display. Depending on their locations, the blue disks could either form a random array, which was essentially irrelevant to the target's location, or a contextual array, which carried a fixed spatial relationship to the target (Figure 1; see also below).

Each of the stimulus items (T, L, and disk) appeared in one of the 120 "cells" (each cell indicated by a cross marker, Figure 1), evenly distributed over an area that subtended $23.1^{\circ} \times 23.1^{\circ}$ of visual angle. The 120 cells were equally divided into eight parts (octants), each containing 15 cells. To ensure a spatially even distribution of the stimulus items, we contrived a rule for the allocation of each item. First, four different octants were used for the target location for the cued displays, and the remaining four were used for the random displays. Within each octant, there was only one possible target location (Figure 1). Second, for both cued and random displays, each of the eight cue elements was located within an octant. Third, the number of the target and distractors combined was equal in each octant, with the exact locations of distractors randomly chosen therein (Figure 1). There were thus four different cued displays, repeatedly presented throughout the experiment. The cue items of a random display could appear at any of the 120 cell locations except those allocated to the target, and no random display repeated itself across trials.

There were a total of 576 trials in the experiment, which was divided into six consecutive epochs. There were three different set sizes - 16, 24, and 32-with each set size repeated 8 times for a cued display and 24 times for a random display during each epoch. There were thus three times as many random as cued displays in the experiment. In addition to the one target and the eight cue elements, displays with set size 16,24 , and 32 contained 7, 15, and $23 \mathrm{~L}$ distractors, respectively. To ensure that learning could proceed equally fast for the three set sizes, for each epoch trials with the cued display for a particular set size could be presented only after at least an equal number of such trials for the other set sizes had been presented. Otherwise, trials with cued and random displays were presented in a pseudorandom sequence. 
(a) Spatial arrangement of display

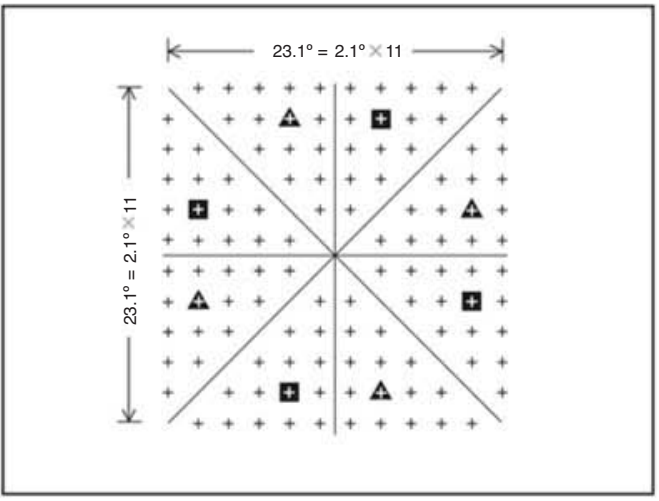

(b) Cued (or contextual) display

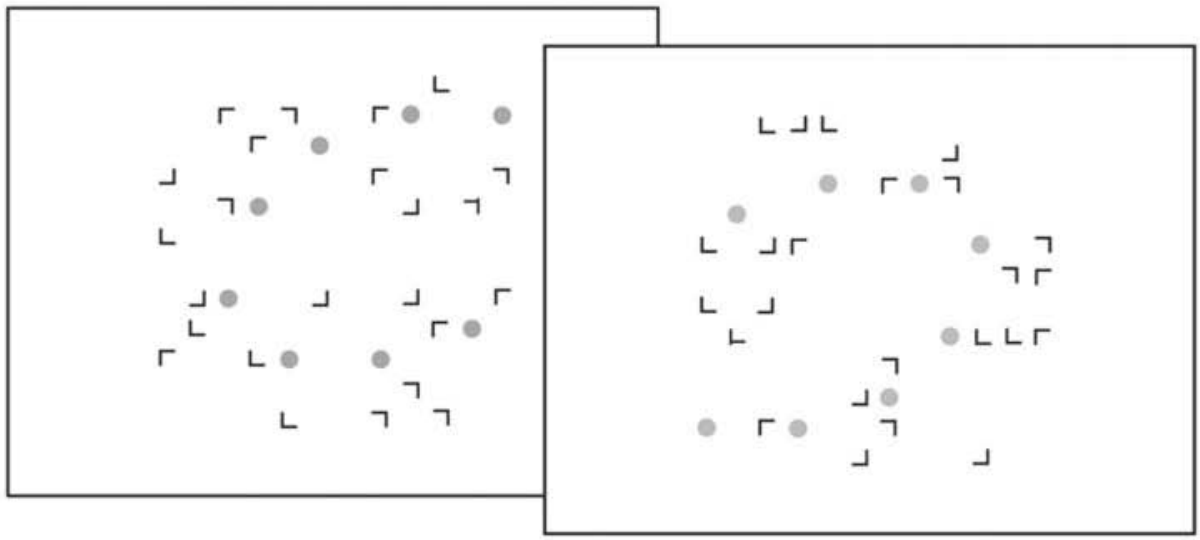

(c) Random display

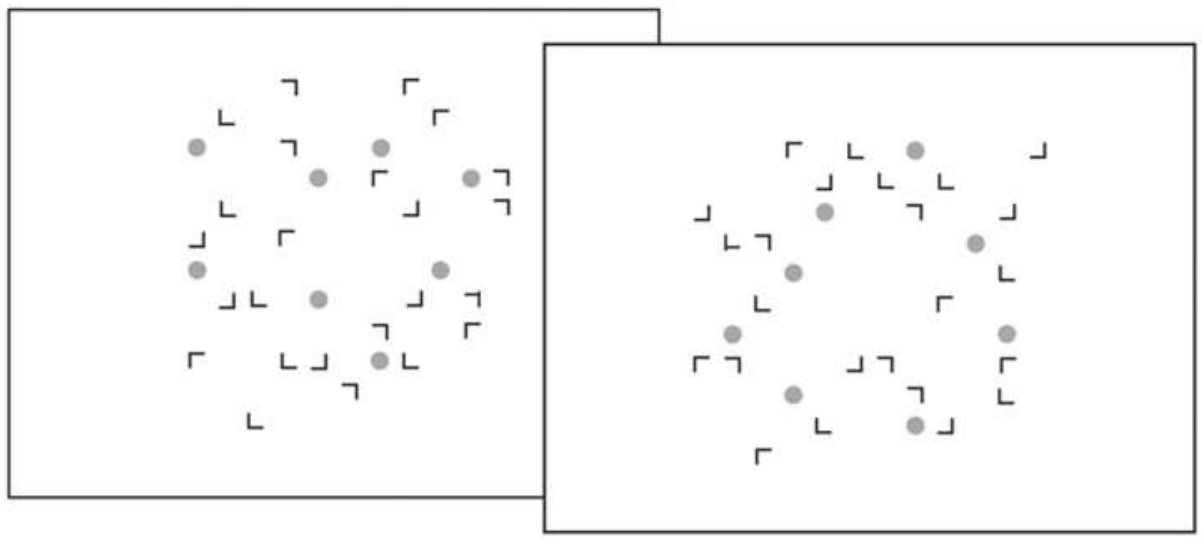

Figure 1. Stimulus display. (a) There were 120 possible spatial locations within a square approximately $23^{\circ} \times 23^{\circ}$ of visual angle, where the stimulus items could appear. Each of these locations is indicated by a cross marker. The display area was evenly divided into eight octants, each housing 15 possible locations. Each potential location of the target was indicated by a triangle (four for cued display) and a square (four for random display). For both the cued (or contextual) and random arrays, each of the eight cue elements was located within a different octant. Equal numbers of the target $T$ and distractors Ls together were positioned in each octant, with the exact locations of distractors randomly chosen therein. Eight cue elements form either a cued (b) or a random (c) array (two examples are shown for each). In the contextual or cued display, the spatial relationship between target and the cued array is constant: The target is always located close to the "opening" of the array even though the array may be oriented differently from trial to trial, as seen in the two examples in the figure. In the random display, there is no fixed spatial relationship between the target and the random array. 


\section{Experimental Procedures}

The subjects were instructed to relax and be seated with their head supported by a chinrest. Each trial began with the appearance of a center light dot, which the subjects were instructed to fixate. After the subject acquired fixation of the center light within a spatial window of a circle $2.24^{\circ}$ in diameter and for $500 \mathrm{msec}$, a search display came on and remained visible until either a button was pressed to signal a judgment or when $10 \mathrm{sec}$ had elapsed, whichever came sooner. The subjects were instructed to be as accurate and as fast as possible in their search of the target $\mathrm{T}$. The subjects pressed one of two buttons to signal the direction (right or left) that the target $\mathrm{T}$ pointed. No mention was made or clues were given as to the meaning of the blue disks. A practice session with 20 trials of random displays was run for each subject before the experiment proper. A 15 -sec break was imposed after every two blocks.

When the subjects finished the search experiment, they were shown two displays, one with a cue and the other with a random array. They made a forced choice of the display they were more familiar with and indicated whether they chose with confidence or by guessing. The entire experiment lasted less than $60 \mathrm{~min}$ for all subjects.

\section{Data Analysis}

Error trials were not included in further analyses. We first examined the mean search time across epochs. As different individuals might exhibit a different rate of learning, we computed for each subject the average reaction time across epochs, each for the three set sizes and for the two arrays. A learning effect resulting from contextual cuing was demonstrated by a greater decrease of reaction time for the cued than for the random display across the six epochs. For each subject, we examined across epochs and determined a cutoff point when such an effect began to be evident. The cutoff was determined such that, among all comparisons between cued and random trials, the reaction time decreased to the greatest extent for cued trials in the epoch after the cutoff, compared with that just prior to the cutoff. In other words, we looked for the maximum of $\left[\left(\mathrm{RT}_{c, n+1}-\mathrm{RT}_{c, n}\right)-\left(\mathrm{RT}_{r, n+1}-\mathrm{RT}_{r, n}\right)\right]$, where $c$ and $r$ stand for cued and random trials, respectively, and $n$ and $n+1$ are the epoch number, with $n$ ranging from 1 to 5 . We defined the epochs prior to this time as the prelearning period and those after this time as the postlearning period. This cutoff was determined separately for the three different set sizes.

For the analyses of scan paths, we defined six oculomotor parameters (time from the start of the search display to the occurrence of the first saccade, total number of saccades in a trial, mean saccade amplitude, mean intersaccadic fixation duration, distance between first eye fixation and the target after display onset, time from last eye fixation to buttonpressing). A multiple-regression analysis was performed for each of the set sizes with the learning effect as the dependent variable and the changes in these oculomotor parameters as the independent variables. The learning effect was defined as the reaction time (RT) gain: the difference between the contextually cued (cue) and randomly cued (rnd) trials in the decrease of reaction time from the prelearning period to the postlearning period. Namely, learning effect $=\mathrm{RT}$ gain $=\left[\left(\mathrm{RT}_{\text {post, cue }}-\mathrm{RT}_{\text {pre, cue }}\right)-\right.$ $\left.\left(\mathrm{RT}_{\text {post, rnd }}-\mathrm{RT}_{\text {pre, rnd }}\right)\right]$. Similarly, the change in each of the six parameters is defined as [( parameter $_{\text {post, cue }}-$ parameter $\left._{\text {pre, cue }}\right)$ ( parameter $_{\text {post, rnd }}-$ parameter $\left.\left._{\text {pre, rnd }}\right)\right]$. The regression provided information as to which parameter could best predict the learning effect in the search task. We also performed repeated measures analyses of variance (ANOVAs) to evaluate which of the six oculomotor parameters actually changed between the prelearning and postlearning periods. Two-way ANOVAs were performed with array (cue vs. random) and period (prelearning vs. postlearning) as the withinsubjects variables, each for the three set sizes.

To elucidate the temporal dynamics of the search behaviors, we computed the distance between consecutive eye fixations and the target so as to evaluate whether each saccade was bringing the eye fixation closer to the target - that is, whether the search process was effective or not. An effective search started when eye movements brought each fixation monotonically closer to the target. The period prior to the effective search was defined as an ineffective search. By performing a linear fit of the eye fixations during the effective search, we were able to obtain a slope, which reflected the efficacy of this search phase. We then evaluated whether this efficacy changed from prelearning to postlearning. We also examined the change of the duration of the ineffective phase and of the distance between the target and where "homing-in" started after learning took place. The details of these analyses are presented along with the results in the next section.

\section{RESULTS}

\section{General Performance}

The mean error rate was $0.76 \%$ across all subjects. A three-way repeated measures ANOVA showed that the error rate did not significantly differ between cue conditions $\left[F(1,11)=1.953, M S_{\mathrm{e}}=0.054, p=.19\right]$, prelearning versus postlearning time period $[F(1,11)=1.821$, $\left.M S_{\mathrm{e}}=0.042 ; p=.204\right]$ or set sizes $[F(2,22)=0.098$, $\left.M S_{\mathrm{e}}=0.016, p=.38\right]$. None of the second- or thirdorder interactions were significant (all $p \mathrm{~s}>.2$ ).

Figure 2 shows the change of reaction time across epochs for set size 32 for each of the 12 subjects. It can be seen that although the decrease of search time is not linear and there is some variability across subjects, overall the trend is similar. Seven of the subjects demonstrated the sharpest RT gain after the second epoch, 3 subjects after the first epoch, and 2 after the third epoch. Remarkably, although the cutoffs between prelearning and postlearning periods were chosen independently for set size 24 , they turned out to be exactly the same as in set size 32 in all the subjects. This result may have reflected the success of our design to ensure even sampling of the three set sizes throughout the experiment. We examined all possible cutoffs (i.e., after the first epoch, second epoch, etc.) for set size 16, but there appeared to be no difference between cued and random displays in the change of search time across epochs in any case. However, for the analyses of eye movement parameters described below, we employed the same cutoff for set size 16, in order to compare the results with those of set sizes 24 and 32 . Figure 3 shows the average change of reaction time across subjects for all three set sizes.

Figure 4 illustrates the search slopes for the two display conditions, separately for each learning period. The search slope appears to decrease for cued trials, compared with random trials, after contextual learning occurs. The mean RTs were subjected to univariate three-way repeated measures ANOVA with display (random vs. cued), learning period (pre- vs. post-), and set size $(16,24$, or 32$)$ as the factors. All effects were significant, and there were significant main effects of display $[F(1,11)=38.13$, $\left.M S_{\mathrm{e}}=10,029.89, p<.001\right]$, period $[F(1,11)=43.27$, $\left.M S_{\mathrm{e}}=25,488.18, p<.001\right]$, and set size $[F(2,22)=$ $\left.120.35, M S_{\mathrm{e}}=28,985.43, p<.001\right]$. The two-way interactions were significant for display $\times$ period $[F(1,11)=$ 

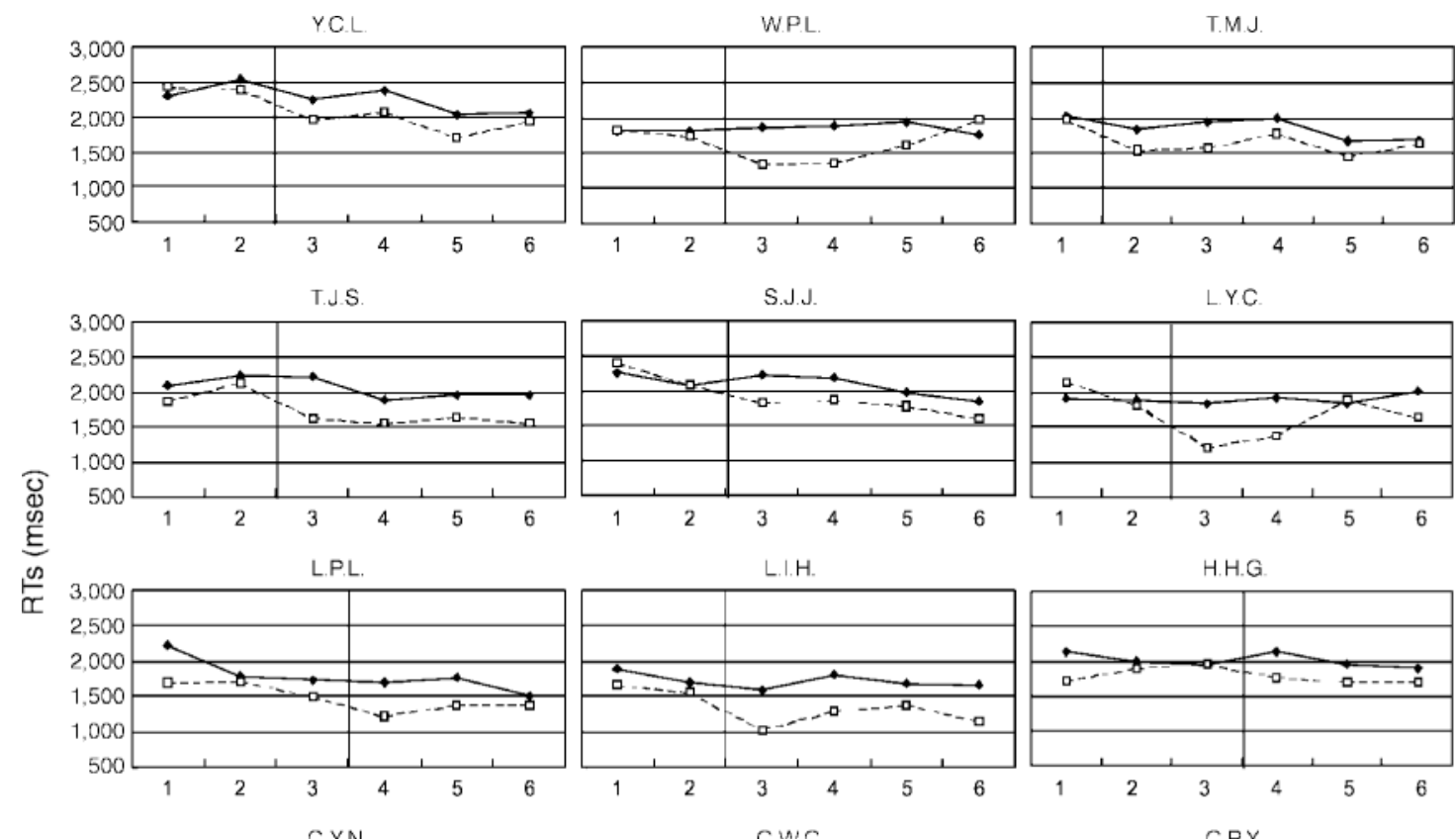

L.I.H.
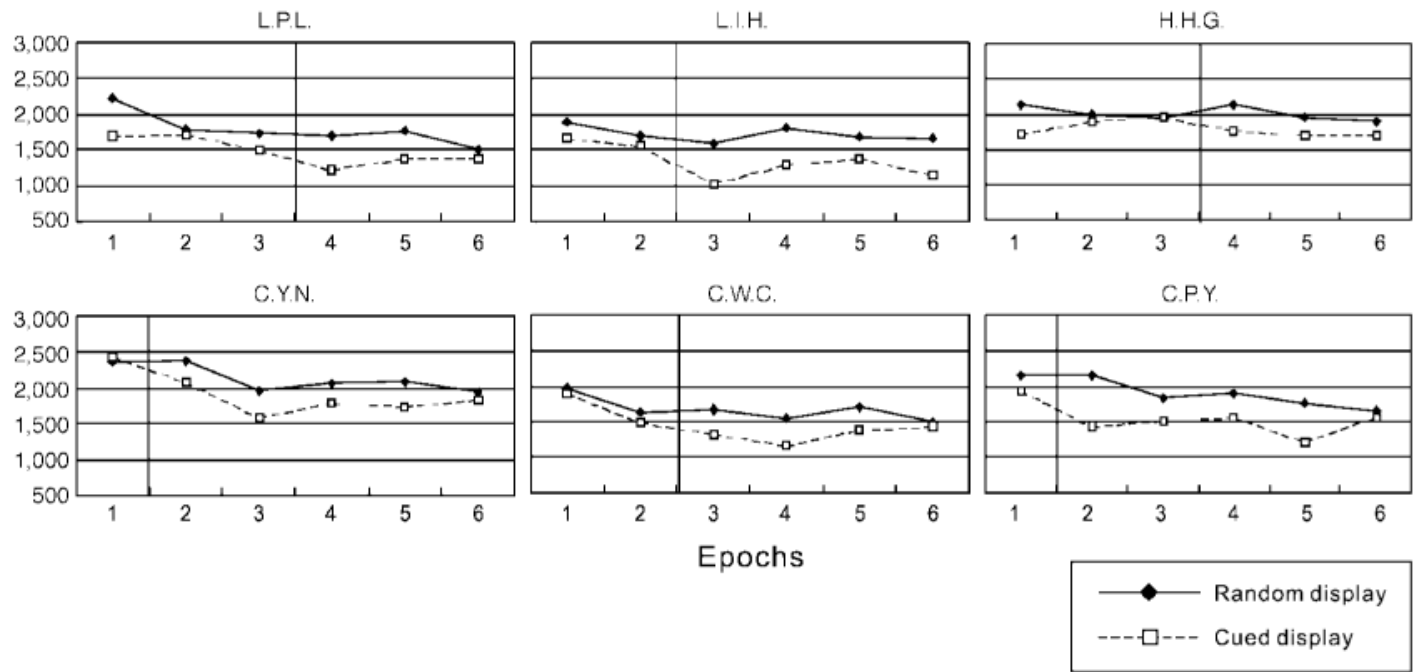

Figure 2. Average search time across epochs for each subject (set size 32). Each data point represents the mean reaction time (RT) within an epoch. The vertical line in each figure delineates the cutoff between prelearning and postlearning periods. For Subjects T.M.J., C.Y.N., and C.P.Y., the sharpest decrease in search time appears after the first epoch. For Subjects L.P.L. and H.H.G., it appears after the third epoch, and for all the other subjects it occurs after the second

$\left.37.31, M S_{\mathrm{e}}=7,202.32, p<.001\right]$, showing a greater learning effect for a cued display, significant for set size $\times$ display $\left[F(2,22)=21.63, M S_{\mathrm{e}}=5,739.5, p<\right.$ $.001]$, showing a greater cue benefit for a larger set size, and significant for set size $\times$ period $[F(2,22)=13.64$, $\left.M S_{\mathrm{e}}=8,545.97, p<.001\right]$, with greater learning for a larger set size. The three-way interaction display $\times$ period $\times$ set size was also significant $[F(2,22)=14.83$, $\left.M S_{\mathrm{e}}=5,381.87, p<.001\right]$.

We next conducted three paired-samples $t$ tests to follow up on the significant three-way interaction to search for any differences between set sizes in the contextual cuing effect. We controlled for a family-wise error rate across these tests using Holm's sequential Bonferroni approach. The results showed that the contextual cuing effect between the two periods was significantly different between set sizes 16 and $32[t(11)=6.39, \alpha=$ $.05 / 3=.0167, p<.001]$ and between set sizes 16 and $24[t(11)=4.29, \alpha=.05 / 2=.025, p<.001]$, but not between set sizes 24 and $32[t(11)=1.32, \alpha=.05 / 1=$ $.05, p=2.14]$.

\section{Explicit Recognition}

The results on the explicit recognition test showed that 7 subjects (all of whom reported guessing) thought that they were more familiar with the cued display, and 5 subjects (all of whom reported guessing) thought that they were more familiar with the random display, which did not significantly differ from chance $(z=1.14, p=.774$, binomial test). None of our subjects reported with confidence their familiarity with the cued display. When asked whether they knew what the blue disks were doing in the visual display, all except 4 subjects reported that they simply ignored them since they looked nothing like the target. Interestingly enough, the other 4 subjects either did not know that there were as many as eight disks in the display or thought that they were present only in very few trials. No one reported taking advantage of the contextual array in the search for the target.

A visual inspection of the eye traces for all subjects showed that none of the eye fixations landed on the blue disks. This finding suggests that the subjects did not overtly attend to the blue disks during their search for the 
(a) Set size 16

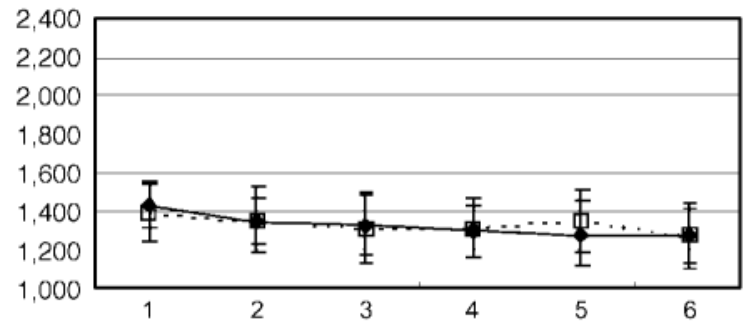

(b) Set size 24

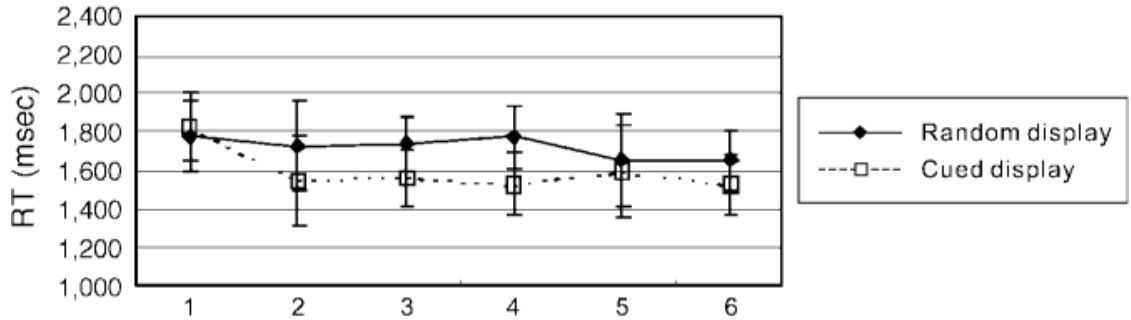

(c) Set size 32

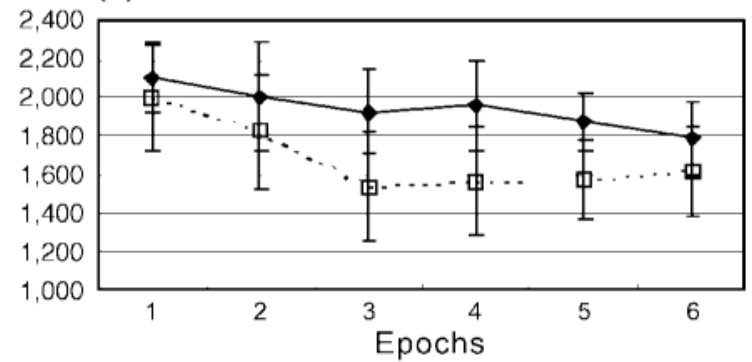

Figure 3. Mean reaction times (RTs) of 12 subjects as a function of learning epoch for the three set sizes. For set sizes 24 and 32, there is a greater decrease in RT across epochs for cued display than for random display. The error bars represent standard deviations of the mean.

target, lending some support to their statements. To corroborate that learning proceeded implicitly, we compared the RT gain between the two groups of subjects who gave opposite answers in the explicit recognition test. The results showed no significant difference between the two groups $\left[F(1,10)=0.274, M S_{\mathrm{e}}=32,727.98, p=\right.$ .61 for set size $24 ; F(1,10)=0.115, M S_{\mathrm{e}}=27,502.92$, $p=.74$ for set size 32; one-way ANOVA]. (a) Prelearning

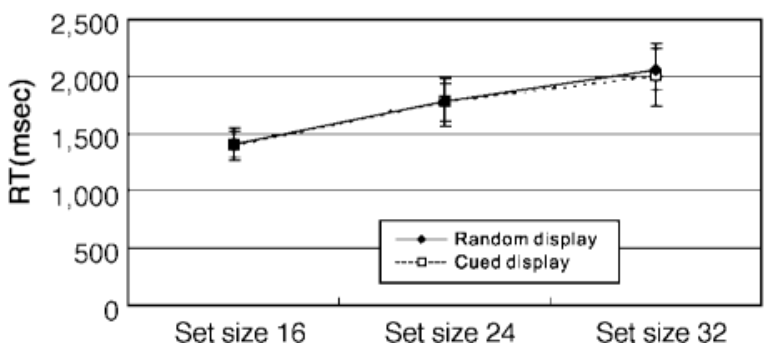

(b) Postlearning

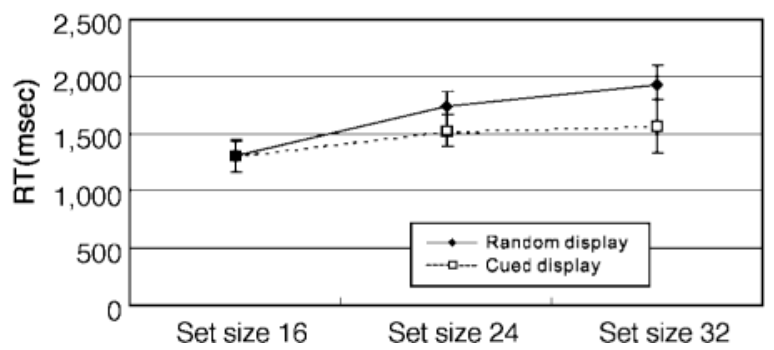

Figure 4. Search slopes of (a) prelearning and (b) postlearning periods. The error bars represent one standard deviation of the mean. In the prelearning period, the search slopes of cued and random trials appear indistinguishable. In the postlearning period, there is a greater reaction time (RT) gain for cued trials for set sizes 24 and 32, but not for set size 16, resulting in a decrease of search slope. 


\section{Which Oculomotor Parameters Accompany Learning in the Search?}

We investigated six oculomotor parameters: Lat (latency), time from the start of the search display to the occurrence of the first saccade; Sacc_Num (saccade number), the total number of saccades in a trial; Amp (amplitude), mean saccade amplitude; Dur (duration), mean intersaccadic fixation duration; D(First Fix, T), distance between the first eye fixation and the target after display onset; and TLFtoBP, time from last eye fixation to button pressing, which presumably would measure the reaction time required for the subject to respond when he or she saw the target. The "last eye fixation" was defined as one of the last two fixations that was spatially closer to the target. This qualification was required because we assumed that target identification occurred at the point when eye fixation was closest to the target, while for a very small number of trials, the chronologically last fixation brought the eye away from the target. Moreover, a minimum of $150 \mathrm{msec}$ was deemed to be required of TLFtoBP. An eye fixation that resulted in a TLFtoBP less than $150 \mathrm{msec}$ was disqualified and replaced by its predecessor.

Tables 1-3 list the mean values of the six oculomotor parameters for cued and random displays in the two periods, each for the three set sizes. For comparison, the RTs are also listed in the tables. Repeated measures ANOVAs showed that, while different parameters varied with respect to learning period or display, only the saccade number came out significant for the interaction as well as for

Table 1

Mean Values of the Six Oculomotor Parameters for Cued and Random Displays in the Two Learning Periods (Set Size 16)

\begin{tabular}{|c|c|c|c|c|c|c|c|c|c|c|c|c|c|}
\hline \multirow[b]{3}{*}{ Measure } & \multicolumn{4}{|c|}{ Learning Period } & \multicolumn{9}{|c|}{ ANOVA Results } \\
\hline & \multicolumn{2}{|c|}{ Prelearning } & \multicolumn{2}{|c|}{ Postlearning } & \multicolumn{3}{|c|}{ Learning Period } & \multicolumn{3}{|c|}{ Display } & \multicolumn{3}{|c|}{ Interaction } \\
\hline & Cued & Random & Cued & Random & $F(1,11)$ & $M S_{\mathrm{e}}$ & $p$ & $F(1,11)$ & $M S_{\mathrm{e}}$ & $p$ & $F(1,11)$ & $M S_{\mathrm{e}}$ & $p$ \\
\hline RT (msec) & 1,408 & 1,410 & 1,312 & 1,308 & 13.252 & 8,858 & .004 & .001 & 4,593 & .97 & .003 & 4,001 & .859 \\
\hline Latency (msec) & 199 & 211 & 206 & 213 & .95 & 217.6 & .351 & 8.712 & 119.2 & .013 & .591 & 145.8 & .458 \\
\hline Saccade number & 5.05 & 5.00 & 4.58 & 4.61 & 17.066 & .129 & .002 & .005 & .102 & .942 & .169 & .079 & .689 \\
\hline Amplitude (deg) & 8.90 & 8.76 & 8.42 & 8.44 & 3.05 & .637 & .109 & 3.246 & .013 & .099 & 1.74 & .05 & .214 \\
\hline Duration (msec) & 199 & 197 & 210 & 210 & 11.764 & 151 & .006 & 2.191 & 11.12 & .167 & 1.459 & 9.83 & .252 \\
\hline D(First Fix, T) (deg)* & 11.78 & 11.5 & 11.75 & 11.6 & .019 & .533 & .893 & .832 & .671 & .381 & .111 & .436 & .745 \\
\hline TLFtoBP $(\mathrm{msec})^{\dagger}$ & 422 & 408 & 374 & 375 & 10.966 & 1,832 & .007 & .543 & 947.2 & .477 & .58 & 1,189 & .462 \\
\hline
\end{tabular}

Note- *Distance between first eye fixation and target; $†$ Time between last eye fixation and buttonpress.

Table 2

Mean Values of the Six Oculomotor Parameters for Cued and Random Displays in the Two Learning Periods (Set Size 24)

\begin{tabular}{|c|c|c|c|c|c|c|c|c|c|c|c|c|c|}
\hline \multirow[b]{3}{*}{ Measure } & \multicolumn{4}{|c|}{ Learning Period } & \multicolumn{9}{|c|}{ ANOVA Results } \\
\hline & \multicolumn{2}{|c|}{ Prelearning } & \multicolumn{2}{|c|}{ Postlearning } & \multicolumn{3}{|c|}{ Learning Period } & \multicolumn{3}{|c|}{ Display } & \multicolumn{3}{|c|}{ Interaction } \\
\hline & Cued & Random & Cued & Random & $F(1,11)$ & $M S_{\mathrm{e}}$ & $p$ & $F(1,11)$ & $M S_{\mathrm{e}}$ & $p$ & $F(1,11)$ & $M S_{\mathrm{e}}$ & $p$ \\
\hline Latency (msec) & 204 & 219 & 216 & 223 & 4.026 & 209.93 & .07 & 11.33 & 125.12 & .006 & 1.643 & 203.06 & .226 \\
\hline Saccade number & 6.42 & 6.32 & 5.33 & 6.21 & 7.358 & .587 & .02 & 10.543 & .166 & .008 & 12.45 & .231 & .005 \\
\hline Amplitude (deg) & 8.62 & 8.69 & 8.30 & 8.26 & 2.934 & .569 & .115 & .114 & .038 & .742 & .558 & .038 & .471 \\
\hline Duration (msec) & $\begin{array}{l}0.02 \\
199\end{array}$ & 199 & 210 & 212 & 10.115 & 172.31 & .009 & .736 & 9.079 & .409 & 1.165 & 7.439 & .303 \\
\hline
\end{tabular}

Note- *Distance between first eye fixation and target; †Time between last eye fixation and buttonpress.

Table 3

Mean Values of the Six Oculomotor Parameters for Cued and Random Displays in the Two Learning Periods (Set Size 32)

\begin{tabular}{|c|c|c|c|c|c|c|c|c|c|c|c|c|c|}
\hline \multirow[b]{3}{*}{ Measure } & \multicolumn{4}{|c|}{ Learning Period } & \multicolumn{9}{|c|}{ ANOVA Results } \\
\hline & \multicolumn{2}{|c|}{ Prelearning } & \multicolumn{2}{|c|}{ Postlearning } & \multicolumn{3}{|c|}{ Learning Period } & \multicolumn{3}{|c|}{ Display } & \multicolumn{3}{|c|}{ Interaction } \\
\hline & Cued & Random & Cued & Random & $F(1,11)$ & $M S_{\mathrm{e}}$ & $p$ & $F(1,11)$ & $M S_{\mathrm{e}}$ & $p$ & $F(1,11)$ & $M S_{\mathrm{e}}$ & $p$ \\
\hline RT (msec) & 2,010 & 2,057 & 1,568 & 7 & 76.65 & 12,839 & .000 & 46.635 & 10,788 & .000 & 46.22 & 6,322 & .000 \\
\hline Latency (msec) & 217 & 213 & 222 & 220 & 1.67 & 258.99 & .223 & .969 & 104.99 & .346 & .087 & 126.56 & .773 \\
\hline Saccade number & 7.10 & 7.39 & 5.29 & 6.71 & 59.25 & .315 & .000 & 54.112 & .256 & .000 & 36.60 & .104 & .000 \\
\hline Amplitude (deg) & 8.46 & 8.50 & 8.27 & 8.30 & .95 & .485 & .351 & .347 & .037 & .568 & .003 & .063 & .958 \\
\hline Duration (msec) & 203 & 201 & 213 & 213 & 9.44 & 162.88 & .011 & 3.598 & 2.88 & .084 & 1.36 & 6.809 & .268 \\
\hline D(First Fix, T) (deg)* & 10.34 & 11.64 & 10.22 & 11.79 & .003 & .778 & .961 & 16.897 & 1.453 & .002 & .35 & .596 & .563 \\
\hline TLFtoBP $(\mathrm{msec})^{\dagger}$ & 413 & 439 & 385 & 410 & 3.62 & 2,764 & .084 & 2.816 & 2,802 & .121 & .002 & 2,508 & .969 \\
\hline
\end{tabular}

Note-*Distance between first eye fixation and target; †Time between last eye fixation and buttonpress. 
the two main effects. For set sizes 24 and 32, the saccade number significantly differed between prelearning and postlearning periods, showing a more pronounced decrease for the cued display (Tables 2 and 3 ). For set size 32 , for instance, there was a mean decrease of $1.81 \mathrm{sac}-$ cades along with a decrease of $442 \mathrm{msec}$ in RT for contextually cued trials, compared with 0.58 saccades and $130 \mathrm{msec}$ for randomly cued trials.

Results from regression analyses revealed whether a decrease in saccade number correlated with a decrease in search time. Note that the values employed in the regression analyses were the changes of each of these parameters resulting from learning through contextual cuing, not through their individual mean values. For set size 16, the regression of the RT gain on the changes in the six oculomotor parameters was significant $\left[R^{2}=.95\right.$; $F(6,5)=15.38, p=.004]:$ RT gain $=1.32$ Lat +189.21 Sacc_Num +7.35 Amp +.43 Dur -13.34 D(First Fix, $\mathrm{T})+.22$ TLFtoBP -19.4 . Moreover, the partial correlation was significant between the change in saccade number and RT gain $[t(10)=6.94, p=.001]$, but not between the other parameter changes and the RT gain. The results indicated that the change in saccade number best correlated with an RT gain. In other words, a smaller saccade number served to index the decrease in search time as a result of contextual cuing.

For set size 24, the regression showed $\mathrm{RT}$ gain $=.83$ Lat + 176.27 Sacc_Num + 37.31 Amp + 5.14 Dur + $2.44 \mathrm{D}$ (First Fix, T) -.15 TLFtoBP +45.39 , which again was highly significant $\left[R^{2}=.98 ; F(6,5)=44.28\right.$, $p<.001]$. The partial correlation was significant between the change in saccade number and RT gain $[t(10)=$ $12.81, p<.001]$, but not between the other parameter changes and the RT gain. The result indicated again that the change in saccade number best correlated with an RT gain for set size 24 . For set size 32 , the regression showed $\left[R^{2}=.93 ; F(6,5)=10.78, p=.01\right] \mathrm{RT}$ gain $=4.45 \mathrm{Lat}+$ 184.66 Sacc_Num + 22.59 Amp + 8.89 Dur + 17.08 $\mathrm{D}($ First Fix, $\mathrm{T})+.61$ TLFtoBP +85.5 , with a significant partial correlation for Lat $[t(10)=4.12, p=.009]$, Sacc_Num $[t(10)=5.06, p=.004]$, and TLFtoBP $[t(10)=$ $2.72, p=.042]$.

Taking all of this together, it appears that a reduction in the number of saccades is best correlated with learning in context-guided visual search. Figure 5 shows the percentage distribution of all of the trials with different saccade numbers for prelearning and postlearning periods. Two findings are noteworthy. First, most of the trials contained a moderate number of saccades. Second, it appears that after context-guided learning occurred, the trials with a higher number of saccades decreased in number, whereas those with a lower number increased. This particular finding replicated Peterson and Kramer (2001a). However, a change in the average number of saccades involved in a trial could mean a number of different things regarding actual changes in search behavior. A critical question concerns the dynamics of the search process.

\section{Ineffective and Effective Search}

We computed the distance between successive eye fixations and the target, D(Fix, T), in order to describe the temporal dynamics of the search behavior. An important factor in this function was the number of saccades involved before the target was reached in a trial. For the following analyses, we considered those saccade numbers that were represented in at least a mean of $5 \%$ of all trials and only those that were represented in all 12 subjects. This left us with saccade numbers ranging from 3 to 10 , which together made up $85.1 \%$ of the trials.

Figure 6 illustrates the mean D(Fix, T) for all these trials, as a function of consecutive fixations. It appears that for either a cued or a random display, prelearning or postlearning, $\mathrm{D}(\mathrm{Fix}, \mathrm{T})$ contained a monotonic phase, in which each successive fixation brought the eye increasingly closer to the target. Moreover, this monotonic phase was obtained no matter how many saccades (of 3 to 10) there were in a trial. We described this monotonic phase of search as an effective search, in contrast to an earlier phase (ineffective search), where saccadic eye movements did not move fixations increasingly closer to the target. Our examination of the scan path showed that the fixations in the ineffective phase occurred randomly throughout the entire display and did not appear to congregate around any specific area.

To delineate the two phases, we set out to determine the beginning of the effective search. We considered a saccade bringing the fixation closer to the target as "valid." In contrast, a saccade directed away from the target was "invalid." Here, we could employ three different methods. First, we defined the beginning of effective search as the earliest time point when every following saccade was valid ("all saccades valid"). Second, we defined the beginning of effective search as the earliest time point when there were three consecutive valid saccades ("three saccades valid"). Third, we defined it as the time when the eye fixation was most distant from the target ("most distant fixation"). The three definitions led to the same results in all but 4 of the 32 cases (Figure 6). We then performed for each condition a linear regression of the fixations in the effective phase for each subject. The slope of the regression reflected the efficacy of effective search, or how fast the successive fixations approached the target.

In light of the finding that a reduction in the number of saccades accompanied learning in context-guided search, an intriguing question was: Did the efficacy of the effective search differ between prelearning and postlearning periods? In other words, did the reduction in saccade number occur in the effective search phase, resulting in a change of efficacy? We considered three possible scenarios to address this issue (Figure 7). First, in the efficacy hypothesis, learning proceeded through an increase of efficacy in the effective search. Namely, the slope of the effective search became sharper in the postlearning period in cued, as compared with random, trials. Second, for the alternative, elimination of ineffec- 
(a) Set size 16

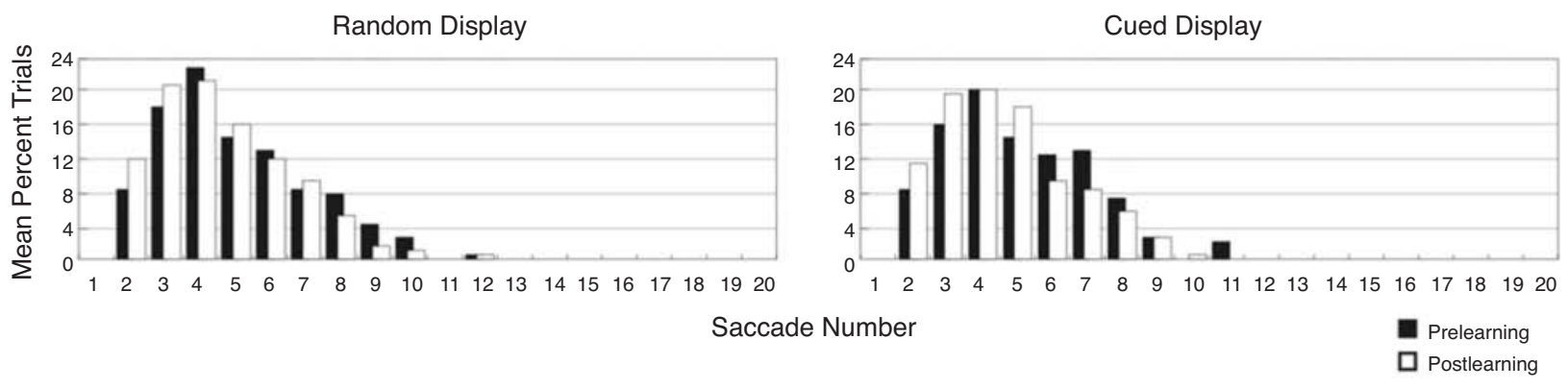

(b) Set size 24

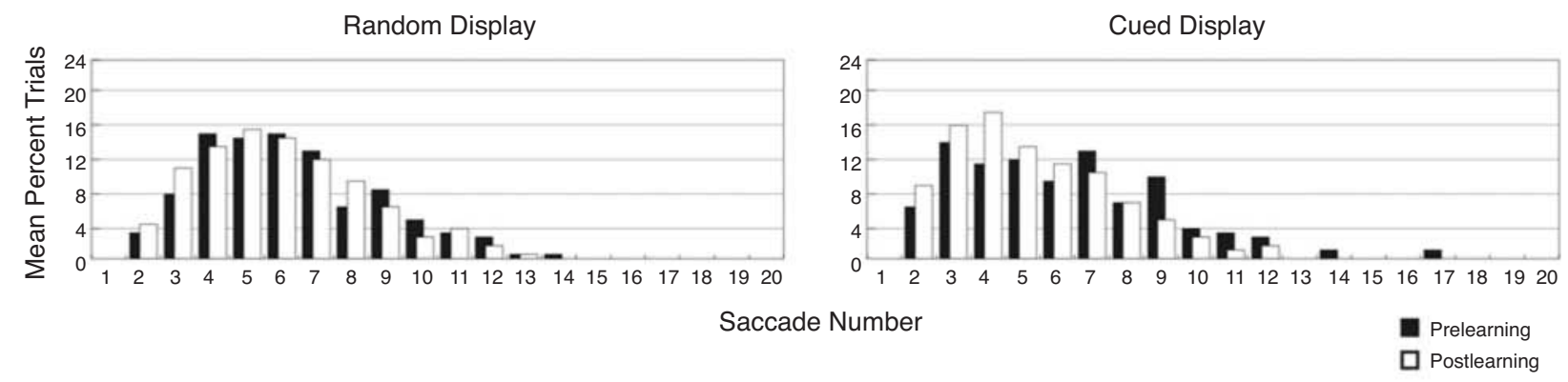

(c) Set size 32

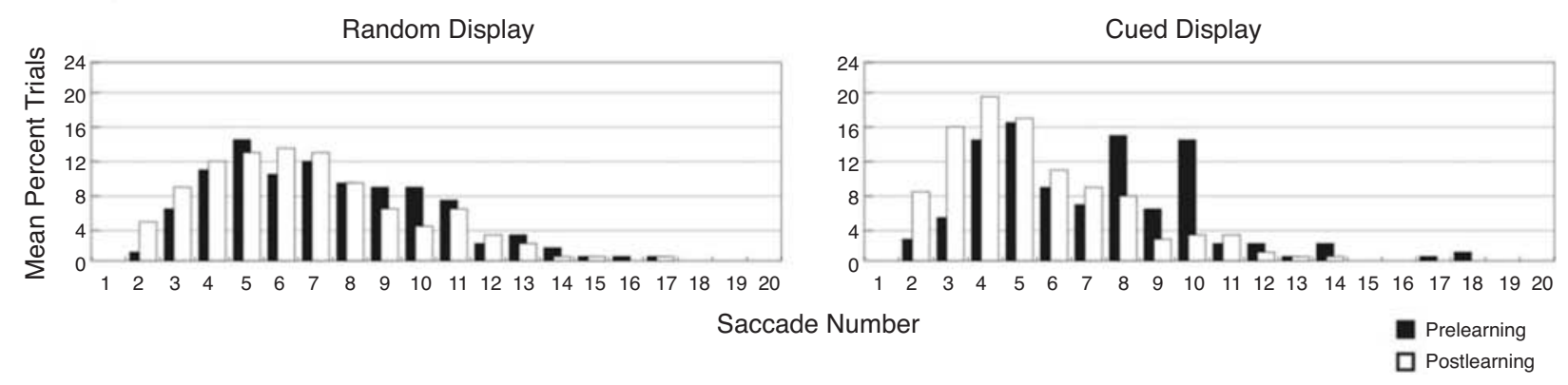

Figure 5. The mean percentages of trials with different saccade numbers in prelearning and postlearning periods. Data are averaged across all subjects. Approximately $85 \%$ of all trials contain 3 to 10 saccades. After learning occurs, the trials with a higher number of saccades decrease, while those with a lower number increase. This change appears to be most conspicuous for set size 32.

tive search hypothesis, a visual search was facilitated by a reduction in the number of saccades in the ineffective search phase. In other words, the slope or efficacy of the effective search phase did not change as a result of contextual cuing. A third hypothesis proposed a mixed mechanism, in which the reduction in saccade number occurred both for the ineffective and effective search phases.

The result obtained from the analysis of saccade number, described above, provided an important clue to help distinguish between these hypotheses. Figure 5 shows that after context-guided learning occurred, the trials with a higher number of saccades decreased in number, whereas those with a lower number increased. Trials with a higher number of saccades involved a longer ineffective phase, whereas those with a lower number involved a shorter phase. The average length of the ineffective period (the number of saccades "wasted") could be computed by taking into account the frequency of trials with different saccade numbers prelearning and postlearning and for cued and random displays (Figure 8A). Since the three algorithms of dividing up the effective and ineffective search phases came to similar results, we adopted "most-distant-fixation" to define the beginning of the effective phase. We then quantified the change of the length of the ineffective phase by performing a repeated measures ANOVA with display and learning period as two factors, separately for the three set sizes. The results showed that, for set size 16 , there was no significant main effect for learning period $\left[F(1,11)=2.787, M S_{\mathrm{e}}=\right.$ $0.114, p=.123]$ or for display $\left[F(1,11)=0.005, M S_{\mathrm{e}}=\right.$ $0.192, p=.944]$, nor was there a significant interaction 
Prelearning (Set size 24)
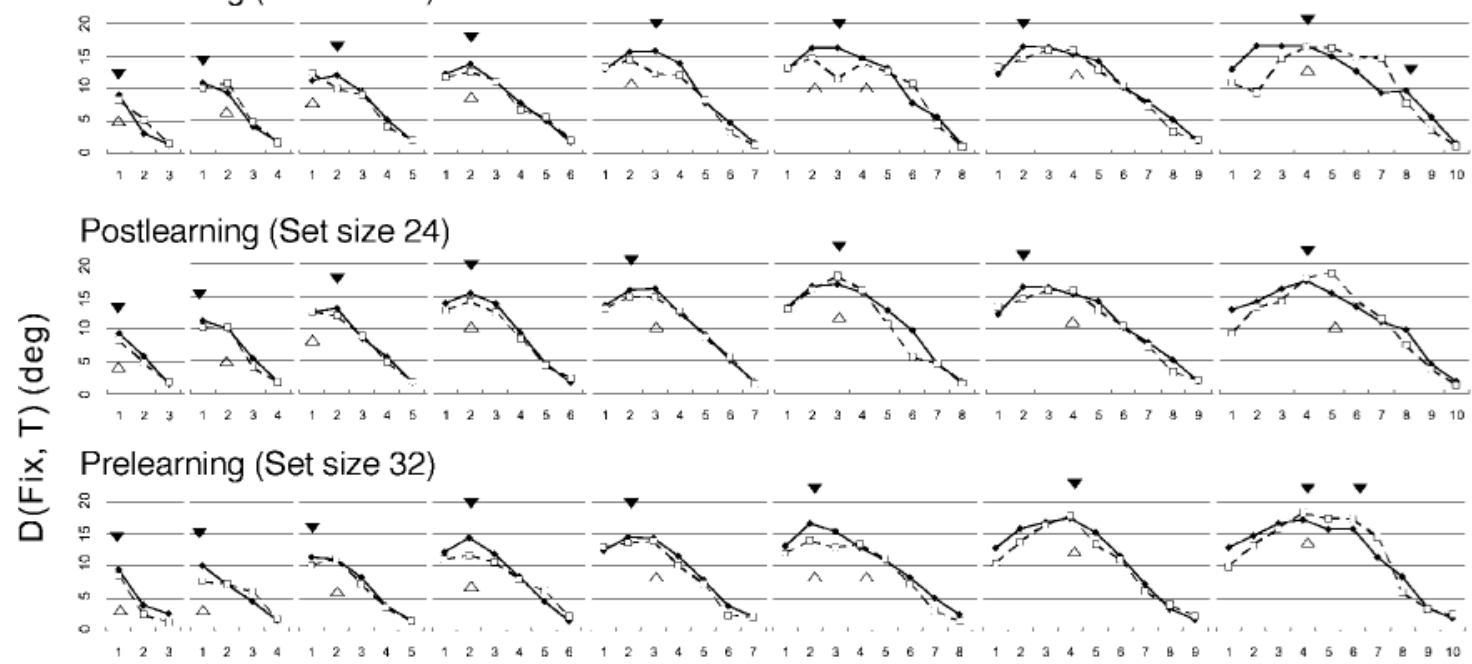

\section{Postlearning (Set size 32)}

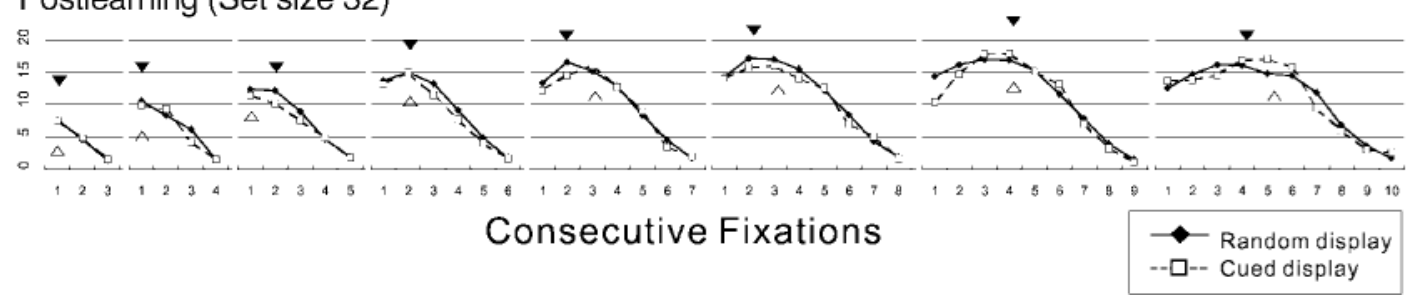

Figure 6. Mean D(Fix, T), or the distance between each eye fixation and the target, as a function of consecutive fixations. Each column shows the plots for trials involving a particular number of saccades (3 to 10). Each data point represents the average of 12 subjects. It is notable that for either cued or random display, prelearning or postlearning, the function appears to comprise two distinct phases: an earlier phase where the eye fixations did not get increasingly closer to the target and a later phase where each successive fixation brought the eye monotonically closer to the target. The solid inverted (for random display) and the open upright (for cued display) triangles represent the start of the effective search phase as defined by the three different methods. The three definitions lead to the same result in all but 4 of the 32 cases (two triangles of the same kind).

$\left[F(1,11)=1.611, M S_{\mathrm{e}}=0.129, p=.231\right]$. For set size 24 , there was a significant main effect for learning pe$\operatorname{riod}\left[F(1,11)=7.394, M S_{\mathrm{e}}=0.027, p=.02\right]$ but not for display $\left[F(1,11)=0.049, M S_{\mathrm{e}}=0.082, p=.828\right]$. Moreover, there was a greater shortening of the ineffective phase postlearning for the cued display, although the difference did not reach statistical significance $[F(1,11)=$ $2.953, M S_{\mathrm{e}}=0.021, p=.114$, period $\times$ display interaction]. Likewise, for set size 32 , there was a significant main effect for learning period $\left[F(1,11)=20.370, M S_{\mathrm{e}}=\right.$ $0.048, p=.001]$ but not for display $[F(1,11)=0.295$, $\left.M S_{\mathrm{e}}=0.181, p=.598\right]$, and a significant interaction $\left[F(1,11)=6.502, M S_{\mathrm{e}}=0.048, p=.027\right]$, showing a greater shortening of the ineffective phase postlearning for the cued display. This analysis overall supported the elimination of the ineffective phase or the mixed mechanism hypothesis.

A decrease in the duration of the ineffective phase should mean an earlier start of the effective, "homingin" process. We thus also examined whether the effective search phase started on average at an earlier time postlearning for the cued display. To this end, we computed the change of the "most distant fixation" (which represented the peak among all distances between the fixations and the target, or peak $\mathrm{D}[\mathrm{Fix}, \mathrm{T}]$ ) prelearning and postlearning and separately for random and cued displays. The ANOVA results corroborated the change of the duration of the ineffective phase (Figure $8 \mathrm{~B}$ ). For set size 16, there was no significant main effect for learning period $\left[F(1,11)=0.128, M S_{\mathrm{e}}=1.493, p=.727\right]$ or display $\left[F(1,11)=1.171, M S_{\mathrm{e}}=1.26, p=.302\right]$. The interaction term was not significant, either $[F(1,11)=0.154$, $\left.M S_{\mathrm{e}}=0.125, p=.702\right]$. For set size 24 , there was a significant main effect for learning period $[F(1,11)=6.804$, $\left.M S_{\mathrm{e}}=0.07, p=.024\right]$ but not for display $[F(1,11)=$ $\left.0.738, M S_{\mathrm{e}}=0.748, p=.408\right]$, and a significant interaction $\left[F(1,11)=12.28, M S_{\mathrm{e}}=0.07, p=.005\right]$, showing a greater shortening of peak $\mathrm{D}(\mathrm{Fix}, \mathrm{T})$ postlearning for the cued display. The effective search phase started at a mean distance of $14.6^{\circ}$ and $14.6^{\circ}$ prelearning, and $14.7^{\circ}$ and $14.2^{\circ}$ postlearning, for random and cued displays, respectively. Likewise, for set size 32 , there was a signif- 
(a) Efficacy hypothesis

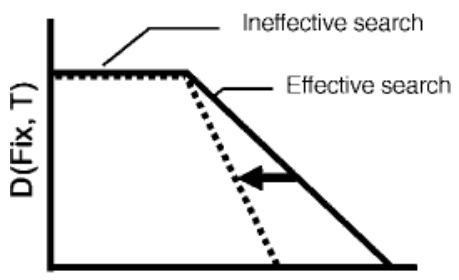

Consecutive Saccade

(b) Elimination of ineffective search hypothesis

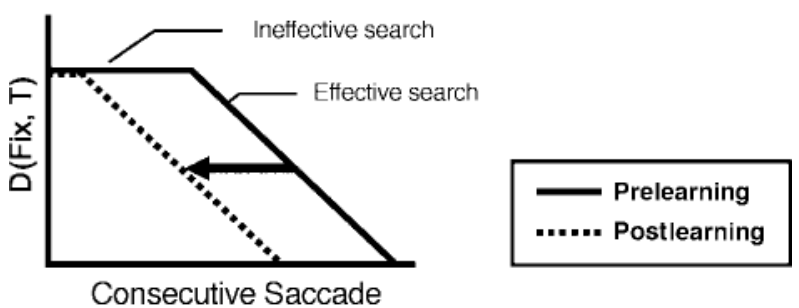

(c) Mixed mechanism

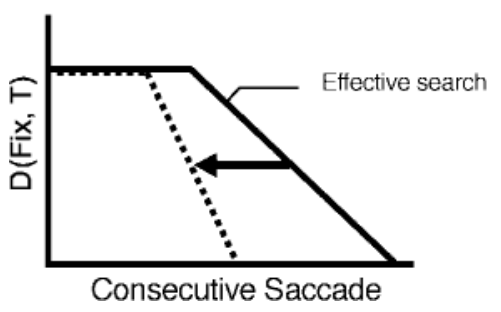

Figure 7. The three hypothetical models of how a reduction in saccade number leads to a decrease in search time after learning occurs. (a) In the efficacy hypothesis, learning proceeds through an increase of efficacy in the effective search. In other words, the slope of the effective search becomes sharper in the postlearning period in cued trials, in comparison with random trials. (b) In the elimination of the ineffective search hypothesis, a visual search is facilitated by a reduction in the number of saccades in the ineffective search phase. The slope or efficacy of the effective search phase does not change as a result of contextual cuing. (c) In the mixed mechanism, the saccade number decreases during both the ineffective and effective search phases.

icant main effect for learning period $[F(1,11)=12.623$, $\left.M S_{\mathrm{e}}=1.181, p=.005\right]$ but not for display $[F(1,11)=$ $\left.1.218, M S_{\mathrm{e}}=1.322, p=.293\right]$, and a significant interaction $\left[F(1,11)=9.26, M S_{\mathrm{e}}=0.763, p=.011\right]$, showing a greater shortening of peak $\mathrm{D}(\mathrm{Fix}, \mathrm{T})$ postlearning for the cued display. The effective search phase started at a mean distance of $14.8^{\circ}$ and $14.4^{\circ}$ prelearning, and $15.2^{\circ}$ and $13.3^{\circ}$ postlearning, for random and cued displays, respectively.

To further distinguish between the three hypotheses, we then focused on the effective search phase. We submitted the slopes to a four-way repeated measures ANOVA with set size, display, learning period, and saccade number as factors. The results (with the "most-distant-fixation" algorithm to define the beginning of the effective search) showed that there was no main effect for any of the four factors [display, $F(1,11)=1.140, M S_{\mathrm{e}}=5.418, p=$ .309 ; saccade number, $F(7,77)=1.779, M S_{\mathrm{e}}=2.452$, $p=.104$; all other $F \mathrm{~s}<1$ ], nor any significant two-way [set size $\times$ saccade number, $F(7,77)=1.238, M S_{\mathrm{e}}=$ $1.905, p=.293$; display $\times$ saccade number, $F(7,77)=$ $1.037, M S_{\mathrm{e}}=3.010, p=.413$; all other $F \mathrm{~s}<1$ ] or higher order interactions [set size $\times$ display $\times$ saccade number, $F(7,77)=1.095, M S_{\mathrm{e}}=1.535, p=.375$; all other $\left.F \mathrm{~s}<1\right]$. The results for the main effects still held even when the insignificant interactions were taken out of the models. Therefore, the speed at which the effective search approached the target did not appear to vary with set size, display, learning period, or saccade number. The analyses based on the other two algorithms to define the beginning of the effective phase provided identical results.

The mean slope turned out to be approximately $3^{\circ}$ per saccade no matter how the beginning of the effective search phase was defined $\left(3.15^{\circ} \pm 0.51^{\circ} / \mathrm{sacc}\right.$, "all saccades valid"; $3.12^{\circ} \pm 0.50^{\circ} / \mathrm{sacc}$, "three saccades valid"; and $3.09^{\circ} \pm 0.51^{\circ} / \mathrm{sacc}$, "most distant fixation"). This result ruled out the efficacy or mixed mechanism hypotheses in mediating the learning effect and left us with the elimination of the ineffective search hypothesis, which suggested that the reduction of saccade number mainly occurred during the ineffective search phase. The result suggests that after a subject learns to employ the contextual cue, the search starts at a point where effective search quickly takes over.

\section{GENERAL DISCUSSION}

\section{General Effects of Contextual Cuing on Search Performance}

Consistent with earlier studies, our subjects demonstrated implicit context-guided learning in visual search. The present results extend previous studies by showing that contextual learning can occur even when the context is composed of salient distractors. Although the subjects were not aware that there was a fixed pattern of cue elements repeatedly presented over the trials, they nevertheless benefited from the invariant context. The reaction time gain did not correlate with the measure of explicit recognition, providing further evidence that learning was implicit. As shown in previous studies, subjects appeared to learn to take advantage of the contextual cue early in the experiment. Ten out of the 12 subjects showed a greater decrease in search time for the contextually cued trials before the third epoch-namely, after only $33 \%$ of the trials had been presented.

The learning effect was most prominent for set size 32 , while it was absent for set size 16, the latter perhaps because of a floor effect in search time (with less stimuli crowding the visual scene, the target could be identified in peripheral vision, thus doing away with the contextual learning effect). Our results showed that there was no difference in learning effect for set sizes 24 and 32. However, a larger variety of different set sizes will 
(a)

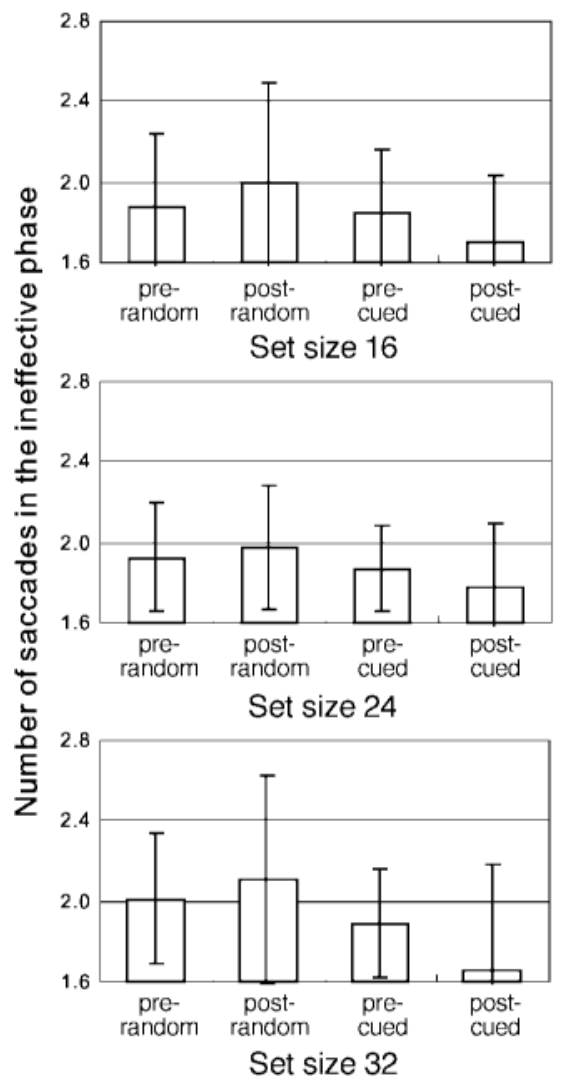

(b)

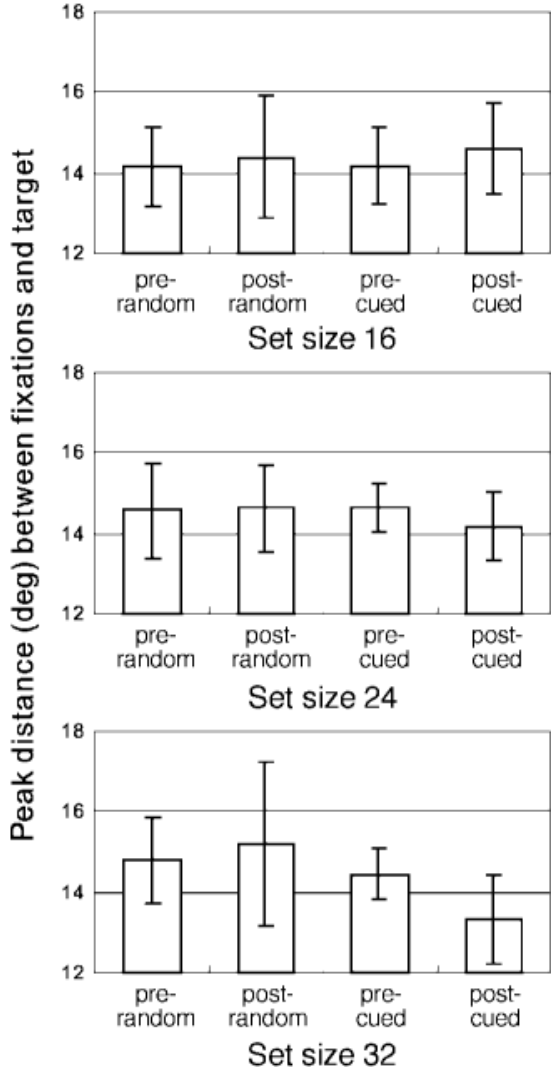

Figure 8. (a) The shortening of the ineffective search phase after contextual learning. The vertical axis represents the number of saccades "wasted" during the ineffective phase. The results are plotted separately for the three set sizes. After learning occurs, the ineffective phase overall becomes shorter, but the effect is much greater for the cued than for the random display (set sizes 24 and 32). (b) Similar results are obtained for the change of peak D(Fix, T) after contextual learning. All plots show the mean of 12 subjects, with the error bar representing standard deviation.

have to be tested before it can be determined whether the effect of implicit learning in context-guided visual search depends on set size (Chun \& Jiang, 1998).

\section{The Role of Attention in Context-Guided Implicit Learning}

The search for the target among distractors in our behavioral task requires serial deployment of attention. A contextual cue improves the search performance, perhaps by enhancing selective attention, as suggested in previous studies (Chun \& Jiang, 1998, 1999; Chun \& Nakayama, 2000). On the other hand, is attention to the contextual cue required for the learning effect to occur? Jiang and Chun (2001) presented evidence that implicit learning of a configuration takes place only when the configuration is selectively attended.

The present results appear to provide a different perspective. The subjects in our experiments reported that they ignored the contextual array in the stimulus display. The observation that none of the eye fixations were on the cue elements was consistent with their subjective reports. In some cases, they were not even aware that the contextual cue was presented in every trial. Nevertheless, they demonstrated implicit learning in the search, with a time course similar to that obtained in earlier experiments. On the other hand, one needs to keep in mind that the finding that subjects did not fixate the cue items may not necessarily suggest that they did not attend to these items. Given the sparseness of the cue items, they may not need to be fixated in order to be attended.

Our results are broadly consistent with the general findings of implicit behavioral changes. For instance, using a negative priming paradigm, DeSchepper and Treisman (1996) demonstrated that implicit memory could develop for novel and meaningless as well as for familiar visual stimuli. The implicit memory for these novel unattended shapes could be established after a single presentation and last as long as a month. It has been suggested that although attention is required to make these memory tokens explicitly or consciously accessi- 
ble, it is not necessary for the memory to be established or consolidated. Our results also agree with those of Hollingworth, Williams, and Henderson (2001), in which subjects' eye movements were monitored as they performed in a change detection paradigm. The latter study demonstrated that whether or not a change in the scene was explicitly detected, the subjects exhibited longer fixation duration on objects involving a change when they refixated on them. This implicit effect could be obtained for an object that was not focally attended when the change occurred.

Although it has been stated that limited attentional resources are involved in implicit learning, empirical studies have largely provided evidence that implicit learning occurs to a lesser extent when attention is not fully available (Cohen, Ivry, \& Keele, 1990; Curran \& Keele, 1993; Stadler, 1995). The cue array in our experiment was composed of items that were distinct in color and shape from the target and distractors and appeared visually salient. On the other hand, our subjects seem to have intentionally ignored these distinct items, which they clearly knew were not the target and might interfere with their search. Since we did not explicitly examine whether and how much the subjects attended to the cue array, we could not directly address the issue of whether attention is required for context-guided learning in visual search or, for that matter, how much attention "spills over" to the irrelevant items in our behavioral paradigm. However, given their subjective reports, we suggest that perhaps endogenous attention does not need to be deployed in this particular form of implicit learning.

\section{Ineffective and Effective Phases in a Serial Search}

It is theorized that the reaction time involved in identifying a target in a serial search task is on average the time required to inspect half of the items in the display. The actual search time varies greatly trial by trial, depending on the number of saccades involved. Some of these eye movements bring the fixation closer to the target, and some bring it away. One interesting finding of the present study is that for most of the trials, the search appears to proceed in an orderly manner. By examining the distance between successive eye fixations and the target, we show that the search process in this behavioral task is composed of two different phases - an ineffective search and an effective search. After an earlier ineffective phase, where saccades are not getting closer to the target, comes an effective phase, where saccades are approaching the target monotonically. The division of search into the two phases can be obtained both in the prelearning and the postlearning periods and both for cued and random trials. For the range of saccade numbers involved in a trial that can be reliably examined, this also appears to be true no matter how many saccades have elapsed before the subject reaches the target.

The above finding is intriguing because a visual search does not have to proceed in this way a priori. One could imagine that the eye fixation moves around each stimulus item during the search and, with the help of a memory process, eventually reaches the target. However, there does not necessarily have to be a monotonic phase anywhere in the search path! With a number of different ways to define the beginning of the effective search, we further quantify its efficacy by computing how fast the target is reached. The efficacy actually does not vary with display (cued vs. random) or saccade number, although trials with a higher number of saccades seem to have a flatter slope (see Figure 6). In other words, for trials that demonstrate the two search phases, the efficacy of the effective search appears to be relatively constant.

To the best of our knowledge, previous studies of visual search that involve eye movement monitoring do not present similar observations. An earlier experiment that explored the scan path of visual search found that eye movements were directed recursively to the centers of the display configuration until the target was acquired (Zelinsky, Rao, Hayhoe, \& Ballard, 1997). Perhaps because of the relatively small set size used in that study, most trials were completed with only three saccades. The scan path that constituted such search appeared to be entirely effective, with each saccade bringing foveation closer to the target. Our study thus replicated and extended this particular finding; a scan path involving more than three saccades tends to be less effective. Given the complexity of visual search, however, further experiments are required to ascertain whether the finding of the two search phases may simply be specific to our behavioral paradigm.

There has been a debate recently about whether memory contributes to visual search (Gibson, Li, Skow, Brown, \& Cooke, 2000; Gilchrist \& Harvey, 2000; Horowitz \& Wolfe, 1998, 2001; Kristjánsson, 2000; Peterson, Kramer, Wang, Irwin, \& McCarley, 2001; Shore \& Klein, 2000). Although the present study does not address this issue directly, the results are consistent with the finding that the locations are checked systematically in a visual search task and that subjects use spatial memory when searching for a target among distractors (Danziger, Kingstone, \& Snyder, 1998; Kristjánsson, 2000; Peterson \& Kramer, $2001 \mathrm{~b})$. Indeed, the mechanism underlying the monotonic approach to the target in the effective search phase could be related to the inhibition of return of attention, as has been suggested previously (Klein \& MacInnes, 1999; Posner \& Cohen, 1984; see Klein, 2000, for a review). Shore and Klein emphasize the importance of memory in a visual search and suggest that between-trials priming might produce some of the benefits that accrue over blocks when consistent mappings are used. In the present study, we demonstrate that subjects benefit from contextual cuing by an earlier entry into an effective search phase when the spatial cue is repeatedly presented throughout the experiment. Implicit memory of the spatial relationship between the cue array and the target carries over from trial to trial. 


\section{Oculomotor Correlates of the Effect of Contextual Cuing on Visual Search}

Consistent with earlier experiments that demonstrate contextual learning in visual search (Chun \& Jiang, 1998; Schneider \& Shiffrin, 1977; Shiffrin \& Schneider, 1977), the present study additionally reveals the oculomotor behaviors that may suggest a mechanism through which learning occurs. We found that, among a number of potential oculomotor parameters, the latency to initiate the first saccade and the distance between the first eye fixation and the target did not change as a result of learning, suggesting that subjects were not deliberately delaying their eye movements in order to land their first eye movement closer to the target. Moreover, the saccade amplitude and the duration of eye fixation are not different, suggesting that subjects were not employing different oculomotor strategies prelearning and postlearning. Finally, the time from the last eye fixation to buttonpress remained unaltered, suggesting that learning does not occur as a result of a change in motor response. In contrast, the reduction in the number of saccades correlates with a greater decrease in search time, when the target is embedded in the same contextual array, than when the target is presented along with a random array.

A recent study by Peterson and Kramer (2001a) employed eye movement recording in a paradigm modified from Chun and Jiang (1998) and found that subjects required fewer fixations to locate a target after contextual learning occurs. In addition, a "recognition" (albeit implicit) mechanism may enable subjects to locate the target with just one fixation in some trials. Our subjects did not report any familiarity with the cue array. Scan-path analysis showed that none of them completed the task with just one fixation. Instead, the reduction in the number of fixations as a result of contextual learning appears to occur after the search has begun, an effect that is evident in trials involving different numbers of saccades. One might surmise that contextual cuing can exert its effect at many different levels of information processing. The effects obtained in our study may be tied to a more "primitive" level, where the cue array is minimally encoded and processed. In contrast, in studies where cue items need to be inspected during the search, more attentional resources are allocated to the context. This in turn would channel the information to a higher level, where a "recognition" mechanism could potentially take place. Another possible explanation for this discrepancy lies in the difference in the visual displays. In the study of Peterson and Kramer (2001a), the contextual information was available before the search items were revealed to subjects, whereas in the present study the contextual array appeared at the same time as the search items. With this information in place early on, the search could be primed such that the recognition mechanism takes attention directly to the target. More experiments are certainly required to explore this issue, but at the very least, the present results are not inconsistent with the findings reported in Peterson and Kramer (2001a).
Further clues to the mechanisms that underlie contextguided learning in our paradigm come from analyses of the temporal dynamics of eye movements. Computation of the distance between successive eye fixations and the target delineates two distinct phases of search behaviorsineffective and effective search. For the present study, the existence of the two search phases provides an opportunity for us to explore the mechanism underlying context-guided learning. Under this conceptual framework, learning could occur solely in the ineffective or effective search phase, or in both phases (see Figure 7).

We can demonstrate that for a majority of trials, the reduction of the saccade number occurs predominantly in the ineffective search phase, where eye movements appear to be "aimless" - not getting closer to the target. Contextual cuing expedites the entry from the ineffective to the effective search phase and thereby decreases the search time, while the efficacy of the effective search does not appear to differ between the prelearning and postlearning periods. This result thus extends the findings of Peterson and Kramer (2001a) by specifying where the reduction in the number of fixations occurs. The result also suggests that the efficacy of the effective search is not amenable to perturbation by contextual factors. However, since a negative finding (in testing for a difference in slope) is not a good indicator of invariance, the results may not indicate that this efficacy reflects an intrinsic operational component of the search process.

Our finding of a reduction postlearning in the duration of the ineffective search phase reminds one of the change of the functional (or useful) field of view (FFOV) investigated in the attention literature (Ball, Beard, Roenker, Miller, \& Griggs, 1988; Pringle, Irwin, Kramer, \& Atchley, 2001; Scialfa, Thomas, \& Joffe, 1994; Seiple, Szlyk, Yang, \& Holopigian, 1996). The FFOV represents the spatial area that is required to successfully perform a visual task without invoking eye or head movements. In these studies, the size of the FFOV of individual subjects is inferred directly with respect to their performance in target identification; a target stands a good chance of being located if it appears within the FFOV. The finding of a speedier entry into the effective search phase after contextual learning occurs is thus analogous to an increase in the size of the FFOV, in the sense that once eye fixation enters this functional field, it begins its monotonic approach to the target. On the other hand, the functional field involved in our contextual search would have to be different from the FFOV involved in these target identification tasks, where eye movements are not allowed or are specifically discouraged. The monotonic approach toward the target within the functional field suggests an order of attentional shift, whose structure deserves further investigation.

Our results demonstrate that context-guided learning in a visual search takes place along with a reduction in the number of saccades. The decrease in saccade number is moderate and appears to be evenly distributed across all trials involving different numbers of eye movements. 
A fair amount of search time continues to be required even after learning occurs, suggesting that learning does not turn a serial search into a parallel search, which mostly involves only one or two saccades (Maioli, Benaglio, Siri, Sosta, \& Cappa, 2001). This result is consistent with the proposal that contextual cuing does not guide attention immediately and exclusively to the target (Chun \& Jiang, 1998). On the other hand, it is worth noting that search time does not differ between set sizes 24 and 32 in the postlearning period (see Figure 4). Therefore, further studies are warranted to determine whether contextual cuing could prime the search in a way that turns a serial process into a parallel process, or transforms one that requires sequential deployment of attention into one that operates preattentively.

In summary, the present study shows that contextual learning in visual search can take place implicitly in a paradigm where the contextual cue is composed of salient items. Learning appears to occur specifically along with a reduction in the number of eye movements during an ineffective search phase, prior to an effective period where eye fixations approached the target monotonically. These findings provide useful information for establishing a conceptual framework to further investigate the mechanism of context-guided visual search.

\section{REFERENCES}

Ball, K. K., Beard, B. L., Roenker, D. L., Miller, R. L., \& GriggS, D. S. (1988). Age and visual search: Expanding the useful field of view. Journal of the Optical Society of America A, 5, 2210-2219.

CAve, K. R. (1999). The feature-gate model of visual selection. Psychological Research, 62, 182-194.

Chun, M. M. (2000). Contextual cueing of visual attention. Trends in Cognitive Sciences, 4, 170-178.

Chun, M. M., \& JiAng, Y. (1998). Contextual cueing: Implicit learning and memory of visual context guides spatial attention. Cognitive Psychology, 36, 28-71.

Chun, M. M., \& Jiang, Y. (1999). Top-down attentional guidance based on implicit learning of visual covariation. Psychological Science, $\mathbf{1 0}$, 360-365.

ChUn, M. M., \& NaKayama, K. (2000). On the functional role of implicit visual memory for the adaptive deployment of attention across views. Visual Cognition, 7, 65-81.

Cohen, A., Ivry, R. I., \& Keele, S. W. (1990). Attention and structure in sequence learning. Journal of Experimental Psychology: Learning, Memory, \& Cognition, 16, 17-30.

Curran, T., \& KeEle, S. W. (1993). Attentional and nonattentional forms of sequence learning. Journal of Experimental Psychology: Learning, Memory, \& Cognition, 19, 189-202.

DANZIGER, S., KInGSTONE, A., \& SNYDER, J. J. (1998). Inhibition of return to successively stimulated locations in a sequential visual search paradigm. Journal of Experimental Psychology: Human Perception \& Performance, 24, 1467-1475.

DeSchepper, B., \& Treisman, A. (1996). Visual memory for novel shapes: Implicit coding without attention. Journal of Experimental Psychology: Learning, Memory, \& Cognition, 22, $27-47$.

Gibson, B. S., Li, L., Skow, E., Brown, K., \& CoOKe, L. (2000). Searching for one versus two identical targets: When visual search has a memory. Psychological Science, 11, 324-327.

Gilchrist, I. D., \& Harvey, M. (2000). Refixation frequency and memory mechanisms in visual search. Current Biology, 10, 12091212.

Henderson, J. M., \& Hollingworth, A. (1999). High-level scene perception. Annual Review of Psychology, 50, 243-271.
Hollingworth, A., Williams, C. C., \& Henderson, J. M. (2001). To see and remember: Visually specific information is retained in memory from previously attended objects in natural scenes. $\underline{\text { Psychonomic }}$ Bulletin \& Review, 8, 761-768.

Horowitz, T. S., \& WolfE, J. M. (1998). Visual search has no memory. Nature, 394, 575-577.

Horowitz, T. S., \& Wolfe, J. M. (2001). Search for multiple targets: Remember the targets, forget the search. Perception \& Psychophysics, 63, 272-285.

JiAnG, Y., \& ChUn, M. M. (2001). Selective attention modulates implicit learning. Quarterly Journal of Experimental Psychology, $\mathbf{5 4}_{\mathbf{4}}$ 1105-1124.

JoNIDES, J., \& YANTIS, S. (1988). Uniqueness of abrupt visual onset in capturing attention. Perception \& Psychophysics, 43, 346-354.

KLEIN, R. M. (2000). Inhibition of return. Trends in Cognitive Sciences, 4, 138-147.

KLeIN, R. M., \& MacInnes, W. J. (1999). Inhibition of return is a foraging facilitator in visual search. Psychological Science, 10, 346352.

KRISTJÁnsson, A. (2000). In search of remembrance: Evidence for memory in visual search. Psychological Science, 11, 328-332.

Maioli, C., Benaglio, I., Siri, S., Sosta, K., \& CaPPA, S. (2001). The integration of parallel and serial processing mechanisms in visual search: Evidence from eye movement recording. European Journal of Neuroscience, 13, 364-372.

OLSON, I. R., \& CHUN, M. M. (2001). Temporal contextual cuing of visual attention. Journal of Experimental Psychology: Learning, Memory, \& Cognition, 27, 1299-1313.

Peterson, M. S., \& KRAMER, A. F. (2001a). Attentional guidance of the eyes by contextual information and abrupt onsets. Perception \& Psychophysics, 63, 1239-1249.

Peterson, M. S., \& Kramer, A. F. (2001b). Contextual cueing reduces interference from task-irrelevant onset distractors. Visual Cognition, 8, 843-859.

Peterson, M. S., Kramer, A. F., Wang, R. F., Irwin, D. E., \& McCarley, J. S. (2001). Visual search has memory. Psychological Science, 12, 287-292.

Posner, M. I., \& Cohen, Y. (1984). Components of visual orienting. In H. Bouma \& D. G. Bouwhuis (Eds.), Attention and performance $X$ : Control of language processes (pp. 531-556). Hillsdale, NJ: Erlbaum.

Pringle, H. L., Irwin, D. E., Kramer, A. F., \& Atchley, P. (2001). The role of attentional breadth in perceptual change detection. Psychonomic Bulletin \& Review, 8, 89-95.

SCHNEIDER, W., \& SHIFFrin, R. M. (1977). Controlled and automatic human information processing: I. Detection, search and attention. Psychological Review, 84, 1-66.

Scialfa, C. T., Thomas, D. M., \& Joffe, K. M. (1994). Age differences in the useful field of view: An eye movement analysis. Optometry \& Vision Science, 71, 736-742.

Seiple, W., Szlyk, J. P., Yang, S., \& Holopigian, K. (1996). Agerelated functional field losses are not eccentricity dependent. Vision Research, 36, 1859-1866.

SHIFFrin, R. M., \& ScHNeIDER, W. (1977). Controlled and automatic human information processing: II. Detection, search and attention. Psychological Review, 84, 127-190.

Shinoda, H., HaYhoe, M. M., \& Shrivastava, A. (2001). What controls attention in natural environments? Vision Research, 41, 35353545 .

Shore, D. I., \& Klein, R. M. (2000). On the manifestations of memory in visual search. Spatial Vision, 14, 59-75.

StadLER, M. A. (1995). Role of attention in implicit learning. Journal of Experimental Psychology: Learning, Memory, \& Cognition, 21, 674-685.

Treisman, A. M., \& Gelade, G. (1980). A feature-integration theory of attention. Cognitive Psychology, 12, 97-136.

Treisman, A. [M.], \& Gormican, S. (1988). Feature analysis in early vision: Evidence from search asymmetries. Psychological Review, 95 , $15-48$.

TuRATTO, M., \& Galfano, G. (2000). Color, form and luminance capture attention in visual search. Vision Research, 40, 1639-1643. 
Wolfe, J. M. (1994). Guided Search 2.0: A revised model of visual search. Psychonomic Bulletin \& Review, 1, 202-238.

Wolfe, J. M. (1998). Visual search. In H. Pashler (Ed.), Attention (pp. 13-73). London: University College London Press.

Wolfe, J. M., \& Cave, K. R. (1990). Deploying visual attention: The guided search model. In A. Blake \& T. Troscianko (Eds.), AI and the eye (pp. 79-103). Chichester, U.K.: Wiley.

YANTIS S., \& JoNides J. (1996). Attentional capture by abrupt onsets: New perceptual objects or visual masking? Journal of Experimental Psychology: Human Perception \& Performance, 22, 1505-1513.

Zelinsky, G. J., RaO, R., Hayhoe, M., \& BallarD, D. (1997). Eye movements reveal the spatio-temporal dynamics of visual search. Psychological Science, 8, 448-453.
Zelinsky, G. J., \& Sheinberg, D. L. (1995). Why some search tasks take longer than others: Using eye movements to redefine reaction times. In J. M. Findlay, R. Walker, \& R. W. Kentridge (Eds.), Eye movement research: Mechanisms, processes and applications (pp. 325336). Amsterdam: Elsevier, North-Holland.

Zelinsky, G. J., \& SheinberG, D. L. (1997). Eye movements during parallel-serial visual search. Journal of Experimental Psychology: Human Perception \& Performance, 23, 244-262.

(Manuscript received April 9, 2003; revision accepted for publication March 2, 2004.) 